Article

\title{
Apoptotic Cells Trigger Calcium Entry in Phagocytes by Inducing the Orai1-STIM1 Association
}

\author{
Deokhwan Kim ${ }^{1,2}\left(\mathbb{D}\right.$, Hyunji Moon ${ }^{1,2}$, Hyeokjin Cho ${ }^{1,2}$, Chanhyuk Min ${ }^{1,2}\left(\right.$, Byeongjin Moon ${ }^{1,2} \mathbb{D}$, \\ Susumin Yang ${ }^{1,2}{ }^{1}$, Juyeon Lee ${ }^{1,2}$, Sang-Ah Lee ${ }^{1,2}{ }^{\oplus}$, Hyunjin Park ${ }^{1,2}$, Dae-Hee Lee ${ }^{3}$, Dongtak Jeong ${ }^{4}$, \\ Gwangrog Lee ${ }^{1,2}$ and Daeho Park ${ }^{1,2, * \mathbb{D}}$
}

1 School of Life Sciences, Gwangju Institute of Science and Technology, Gwangju 61005, Korea; po7322@gist.ac.kr (D.K.); hjmoon311@gm.gist.ac.kr (H.M.); jhj@gm.gist.ac.kr (H.C.); alscksgur@gist.ac.kr (C.M.); byeongjinmoon@gist.ac.kr (B.M.); susuminy@gist.ac.kr (S.Y.); iris260@gist.ac.kr (J.L.); sanga03@gist.ac.kr (S.-A.L.); malchic22@gist.ac.kr (H.P.); glee@gist.ac.kr (G.L.)

2 Center for Cell Mechanobiology, Gwangju Institute of Science and Technology, Gwangju 61005, Korea

3 Department of Marine Food Science and Technology, Gangneung-Wonju National University, Gangneung 25456, Korea; neogene@gwnu.ac.kr

4 Department of Molecular and Life Science, College of Science and Convergence Technology, Hanyang University ERICA Campus, Ansan 15588, Korea; cooljdt@hanyang.ac.kr

* Correspondence: daehopark@gist.ac.kr; Tel.: +82-62-715-2890

Citation: Kim, D.; Moon, H.; Cho, H.; Min, C.; Moon, B.; Yang, S.; Lee, J.; Lee, S.-A.; Park, H.; Lee, D.-H.; et al. Apoptotic Cells Trigger Calcium Entry in Phagocytes by Inducing the Orai1-STIM1 Association. Cells 2021, 10, 2702. https://doi.org/10.3390/ cells10102702

Academic Editor: Mounia Chami

Received: 2 September 2021

Accepted: 8 October 2021

Published: 9 October 2021

Publisher's Note: MDPI stays neutral with regard to jurisdictional claims in published maps and institutional affiliations.

\begin{abstract}
Swift and continuous phagocytosis of apoptotic cells can be achieved by modulation of calcium flux in phagocytes. However, the molecular mechanism by which apoptotic cells modulate calcium flux in phagocytes is incompletely understood. Here, using biophysical, biochemical, pharmaceutical, and genetic approaches, we show that apoptotic cells induced the Orai1-STIM1 interaction, leading to store-operated calcium entry (SOCE) in phagocytes through the Mertk-phospholipase C (PLC) $\gamma 1$-inositol 1,4,5-triphosphate receptor $\left(\mathrm{IP}_{3} \mathrm{R}\right)$ axis. Apoptotic cells induced calcium release from the endoplasmic reticulum, which led to the Orai1-STIM1 association and, consequently, SOCE in phagocytes. This association was attenuated by masking phosphatidylserine. In addition, the depletion of Mertk, which indirectly senses phosphatidylserine on apoptotic cells, reduced the phosphorylation levels of PLC $\gamma 1$ and $\mathrm{IP}_{3} \mathrm{R}$, resulting in attenuation of the Orai1-STIM1 interaction and inefficient SOCE upon apoptotic cell stimulation. Taken together, our observations uncover the mechanism of how phagocytes engulfing apoptotic cells elevate the calcium level.
\end{abstract}

Keywords: efferocytosis; Orai1; STIM1; interaction; Mertk; SOCE; calcium flux

\section{Introduction}

Efferocytosis is a process that removes apoptotic cells generated throughout life in multicellular organisms and is essential for homeostasis and during development [1]. In this process, apoptotic cells are specifically removed by phagocytes through interactions between ligands on apoptotic cells and their receptors on phagocytes [2]. The best-known ligand on apoptotic cells is phosphatidylserine (PS), which is present on the inner leaflet of the plasma membrane in living cells and on the outer leaflet of the plasma membrane in apoptotic cells [3,4]. Phagocytes express two types of engulfment receptors that directly or indirectly bind to PS on apoptotic cells [5]. Tim-4, BAI1, and Stabilin-2, which are also called PS receptors, directly bind to PS on apoptotic cells [6-8], whereas TAM receptors and some integrins sense PS on apoptotic cells through the bridging molecules Gas6 and Mfge8, respectively [9].

The TAM receptors, Tyro3, Axl, and Mertk, comprise a unique family of receptor tyrosine kinases (RTKs). This family regulates various cellular processes, including cell proliferation, adhesion, migration, and efferocytosis [10]. The function of Mertk in efferocytosis in the retina and testis is well-established [11-13]. Mertk contains two Ig and two fibronectin type III domains located in its extracellular region and a kinase domain 
located in its cytosolic tail. The Ig domains of Mertk associate with Gas6, which binds to PS on apoptotic cells through its Gla domain, thereby promoting phagocytosis of apoptotic cells [14]. The kinase domain of Mertk is also important for efferocytosis because a Mertk mutant lacking this domain fails to promote engulfment of apoptotic cells [15]. In addition, apoptotic cell stimulation induces phosphorylation of Mertk and phospholipase C (PLC) $\gamma 2$ and the association of these two proteins. These suggest that Mertk can transduce signals via its kinase domain and PLC $\gamma 2$ during efferocytosis [16]. However, signal transduction downstream of Mertk during efferocytosis is incompletely understood.

Calcium is involved in a remarkably diverse array of cellular processes in which it functions as a second messenger during signal transduction. Due to its critical roles, the intracellular level of calcium is tightly regulated by various calcium channels and intracellular calcium stores, such as the endoplasmic reticulum (ER) and mitochondria $[17,18]$. One central mechanism regulating the intracellular calcium level is store-operated calcium entry (SOCE), which is mediated by Orai1, a calcium release-activated channel (CRAC), and STIM1, a calcium sensor in the ER. Depletion of calcium in the ER causes STIM1 to accumulate at ER-plasma membrane junctions, where it associates with and activates Orai1, which induces extracellular calcium entry though Orai1 $[19,20]$. Orai1 is typically activated by activation of $\mathrm{G}$ protein-coupled receptors or RTKs that activate PLC to cleave phosphatidylinositol 4,5-bisphosphate ( $\left.\mathrm{PIP}_{2}\right)$ into inositol 1,4,5-triphosphate $\left(\mathrm{IP}_{3}\right)$, which induces $\mathrm{IP}_{3}$ receptor $\left(\mathrm{IP}_{3} \mathrm{R}\right)$-mediated calcium release from the ER [21]. Similar to other cellular processes, calcium is essential for efferocytosis, and its level is modulated for efficient efferocytosis. Thus, inhibition or deficiency of genes involved in calcium flux abrogates efferocytosis [22-24]. However, the molecular mechanism by which apoptotic cells modulate calcium flux in phagocytes remains elusive.

In this study, we found that apoptotic cell stimulation induced the Orai1-STIM1 association in phagocytes. This association was attenuated by masking PS on apoptotic cells, but not by blocking internalization or degradation of apoptotic cells. We further found that apoptotic cell stimulation augmented the phosphorylation of PLC $\gamma 1$ and $\mathrm{IP}_{3} R$. However, this phosphorylation was weakened, and the Orai1-STIM1 association upon apoptotic cell stimulation was attenuated in Mertk ${ }^{-/}$bone marrow-derived macrophages (BMDMs), leading to reduced calcium entry into phagocytes. Collectively, our observations suggest that apoptotic cells induce the Orai1-STIM1 association through the Mertk-PLC $\gamma 1-\mathrm{IP}_{3} \mathrm{R}$ axis, triggering SOCE and elevation of the calcium level in phagocytes during efferocytosis.

\section{Materials and Methods}

\subsection{Plasmids and Antibodies}

All DNA constructs were generated by a PCR-based method and sequenced to confirm their fidelity. Orai1 and STIM1 were amplified from Orai1 (MMM1013-20276444), and STIM1 (MMM1013-202764946) cDNA purchased from Open Biosystems and introduced into pEBB vectors. For Orai1-CFP and STIM1-YFP vector construction, CFP and YFP were amplified from Raichu-Rac1 [25] and C-terminally introduced into pEBB-Orai1 and pEBB-STIM1, respectively. Anti-Flag (Sigma, F1804, St. Louis, MO, USA), anti-Orai1 (Santa Cruz, sc-68895, Dallas, TX, USA), anti-Orai1 (Abcam, ab111960, Cambridge, UK), anti-STIM1 (Abcam, ab108994), anti$\mathrm{IP}_{3} \mathrm{R}$ (Cell Signaling, \#8568, Boston, MA, USA), anti-phospho-IP ${ }_{3} \mathrm{R}$ (Cell Signaling, \#3760S), anti-PLC $\gamma 1$ (Cell Signaling, 2822S), anti-phospho-PLC $\gamma 1$ (Cell Signaling, 2821S), anti-Mer (R\&D systems, AF591, Minneapolis, MN, USA), and anti- $\beta$-Actin (Santa Cruz, sc-1616) were purchased.

\subsection{Cell Culture and Transfection}

LR73 cells were maintained in alpha-MEM containing 10\% fetal bovine serum (FBS) and $1 \%$ penicillin-streptomycin-glutamine (PSQ). BMDMs were maintained in RPMI containing 10\% L929-conditioned medium, 10\% FBS, and 1\% PSQ. NIH3T3 and RAW264.7 cells were maintained in DMEM containing 10\% FBS and 1\% PSQ. LR73 cells were transfected 
with plasmids using Lipofectamine 2000 (Invitrogen, Waltham, MA, USA) or Fugene HD (Promega, Madison, WI, USA), according to the manufacturer's instructions.

\subsection{Mice}

Mertk ${ }^{-/-}$mice were purchased from Jackson laboratory. C57BL/ 6 were purchased from Taconic bioscience. Approximately 8 10-week-old mice were used for experiments regardless of sex. All experiments using mice were approved by the animal care and ethics committees of the Gwangju institute of science and technology (GIST) in accordance with the national institutes of health guide for the care and use of laboratory animals.

\subsection{Binding and Internalization Assay}

BMDMs plated on a cover glass in a 12-well non-culture plate were stained with $0.5 \mu \mathrm{M}$ CellTracker (Thermo, C7025, Waltham, MA, USA) and then incubated with TAMRA-stained apoptotic thymocytes at $4{ }^{\circ} \mathrm{C}$ for $1 \mathrm{~h}$ in the presence or absence of calcium. After that, the cells were washed with PBS and fixed with $4 \%$ PFA. To measure the effect of extracellular calcium on internalization of apoptotic cells, CellTracker-stained BMDMs were incubated with pHrodo-labeled apoptotic thymocytes at $4{ }^{\circ} \mathrm{C}$ for $1 \mathrm{~h}$. After that, unbound apoptotic cells were removed by PBS washing, and the BMDMs were further incubated at $37^{\circ} \mathrm{C}$ for $30 \mathrm{~min}$ in the presence or absence of $\mathrm{Ca}^{2+}$. Images were acquired using Axio Imager D2 (Zeiss, Jena, Germany).

\subsection{Measurement of Intracellular Calcium}

LR73, NIH3T3, RAW264.7, and BMDMs were stained with $1 \mu$ M Fluo4-AM (Thermo, F14201, green) in staining buffer (10 mM HEPES, $150 \mathrm{mM} \mathrm{NaCl}, 5 \mathrm{mM} \mathrm{KCl}, 0.1 \%$ glucose, $1 \% \mathrm{FBS}, 2.5 \mathrm{mM}$ probenecid, $1 \mathrm{mM} \mathrm{CaCl}, 1 \mathrm{mM} \mathrm{MgCl}_{2}$, and $\mathrm{pH} \mathrm{7.4)}$ at $37^{\circ} \mathrm{C}$ for $30 \mathrm{~min}$, pretreated with 10 200 $\mu \mathrm{M}$ 2-APB (Tocris Bioscience, 1224, Minneapolis, MN, USA), $20 \mu \mathrm{M}$ nifedipine (Sigma, N7634), $50 \mu$ M SKF-96365 (Sigma, S7809), or $100 \mu$ M YM58483 (Sigma, Y4895) and incubated with TAMRA-stained apoptotic thymocytes in the presence of the indicated concentrations of the inhibitors for $10 \mathrm{~min}$. Then, the cells were analyzed by flow cytometry (BD FACS Canto II).

\subsection{Engulfment Assay}

Engulfment assay was performed as previously described [26]. LR73 cells or BMDMs derived from the indicated mice were incubated with TAMRA-stained apoptotic thymocytes for $2 \mathrm{~h}$ or $30 \mathrm{~min}$, respectively. Then, the cells were extensively washed with PBS to remove unbound apoptotic cells, trypsinized, suspended, and analyzed by flow cytometry (BD FACS Canto II). TAMRA-positive phagocytes were considered phagocytes engulfing apoptotic cells. To evaluate the effects of inhibitors on efferocytosis, BMDMs were pre-incubated with $10 \mathrm{mM}$ EGTA, 10 200 $\mu \mathrm{M}$ 2-APB (Tocris Bioscience, 1224), $20 \mu \mathrm{M}$ Nifedipine (Sigma, N7634), $100 \mu$ M YM58483 (Sigma, Y4895), $1 \mu$ M Cytochalasin D (Sigma, C8273), $50 \mu \mathrm{M}$ SKF-96365 (Sigma, S7809) or $1 \mu \mathrm{M}$ Bafilomycin A (Abcam, ab120497) for $30 \mathrm{~min}$ (BMDMs) or $2 \mathrm{~h}$ (LR73 cells), and then the engulfment assay was performed.

\subsection{Immunoblotting and Immunoprecipitation}

LR73 cells transfected with the indicated plasmids or BMDMs were incubated with apoptotic cells for $10 \mathrm{~min}$ and then lysed using lysis buffer (50 mM Tris (pH 7.6), $150 \mathrm{mM}$ $\mathrm{NaCl}, 10 \mathrm{mM}$ NaPP, $10 \mathrm{mM} \mathrm{NaF}, 1 \mathrm{mM} \mathrm{Na} \mathrm{VO}_{4}, 1 \%$ Triton X-100, $10 \mu \mathrm{g} / \mathrm{mL}$ pepstatin, $10 \mu \mathrm{g} / \mathrm{mL}$ leupeptin, $10 \mu \mathrm{g} / \mathrm{mL}$ AEBSF, and $10 \mu \mathrm{g} / \mathrm{mL}$ aprotinin). Proteins in the lysates were separated by SDS-PAGE, transferred onto a nitrocellulose membrane, and detected by appropriate antibodies. For immunoprecipitation, the lysates of cells were incubated with anti-FLAG conjugated-agarose beads (Sigma, A2220), glutathione-sepharose 4B beads (GE healthcare, 17-0756-01, Boston, MA, USA), or protein A/G agarose beads (Santa Cruz, sc-2003) with appropriate antibodies for $2 \mathrm{~h}$. Proteins bound to the beads were detected by immunoblotting. ImageJ was used to quantify band intensities. 


\subsection{FRET Analysis for Orai1-STIM1 Association}

LR73 cells plated on confocal dishes were transfected with Orai1-CFP and STIM1-YFP. One day after transfection, the cells were stimulated with TAMRA-stained apoptotic cells for the indicated duration, and then the cells expressing both Orai1-CFP and STIM1-YFP were observed. FRET images were obtained every $10 \mathrm{sec}$ with the ratio imaging method equipped with Olympus FV1c000 SPD (Olympus, Tokyo, Japan). For FRET ratio calculation, CFP and YFP images were subtracted with respective background intensity calculated by the mean intensity value of pixels inside the background mask. The FRET ratio was calculated as the ratio between processed YFP and CFP images. The FRET ratio was presented as a heatmap by mapping the FRET ratio to color and total intensity to opacity. All processing was performed, using custom code of MATLAB R2019b and image processing toolbox. To measure FRET using a microplate reader (FlexStation 3, Molecular devices, San Jose, CA, USA), LR73 cells plated in a 96-well culture plate were transfected with Orai1CFP and STIM1-YFP. One day after transfection, the cells were stimulated with apoptotic or live thymocytes. The FRET values were measured every $10 \mathrm{~s}$ by the microplate reader.

\subsection{SOCE}

BMDMs derived from WT or Mertk ${ }^{-/-}$were plated in a 96-well culture plate and stained with Fluo4-AM (Thermo, F14201, green) in staining buffer (10 mM HEPES, $150 \mathrm{mM}$ $\mathrm{NaCl}, 5 \mathrm{mM} \mathrm{KCl}, 0.1 \%$ glucose, $1 \% \mathrm{FBS}, 2.5 \mathrm{mM}$ probenecid, $1 \mathrm{mM} \mathrm{CaCl} 2,1 \mathrm{mM} \mathrm{MgCl}$, and $\mathrm{pH}$ 7.4). The phagocytes were treated with $0.1 \mu \mathrm{M}$ thapsigargin (Sigma, T9033) for the indicated duration. After that, apoptotic cells or live cells in RPMI containing $1.0 \mathrm{mM}$ calcium were added to the phagocytes at the indicated time. Note that $0.1 \mu \mathrm{M}$ thapsigargin and $1.0 \mathrm{mM}$ calcium were used. The intensity of fluorescence from the cells was measured using a microplate reader (FlexStation 3, Molecular Devices). The peak of SOCE is the maximal fluorescence of Fluo4. Slope is (Fmax - F360s)/(ta - t360s), where ta is the time value at which the fluorescence of Fluo4 is maximal.

\subsection{Statistical Analysis}

Data are shown as the mean \pm standard error of mean (SEM). All experiments were independently performed at least 3 times. Statistical significance of difference was analyzed by unpaired Student's two-tailed $t$ test, one-way ANOVA, or two-way ANOVA, using GraphPad Prism 7. A $p$ value of $<0.05$ was considered to be statistically significant.

\section{Results}

\subsection{Extracellular Calcium Is Required for Internalization of Apoptotic Cells}

Both extra- and intracellular calcium are essential for efferocytosis; however, the reasons why are incompletely understood. Calcium is necessary for binding of PS to its receptors [27-29]; therefore, it is possible that extracellular calcium is crucial for recognition and engulfment of apoptotic cells by phagocytes. We confirmed this hypothesis. Phagocytosis of apoptotic cells by BMDMs treated with EGTA or incubated in calcium-free medium was drastically diminished (Figure 1A), which was likely because apoptotic cells did not bind to them well (Figure 1B,C). However, it is uncertain whether extracellular calcium is solely required for recognition of apoptotic cells by phagocytes. To investigate this, BMDMs were allowed to bind to apoptotic cells without internalization by incubation at $4{ }^{\circ} \mathrm{C}$ and then incubated at $37^{\circ} \mathrm{C}$ in the presence or absence of calcium. Phagocytes incubated in the presence of calcium engulfed apoptotic cells, whereas phagocytes incubated in the absence of calcium bound to, but engulfed few, apoptotic cells (Figure 1D,E). These data suggest that extracellular calcium is required for other stages of efferocytosis following binding of apoptotic cells to phagocytes, implying that it enters phagocytes. 

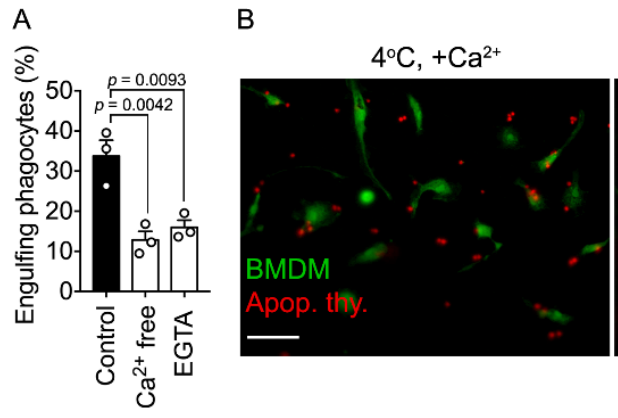

D

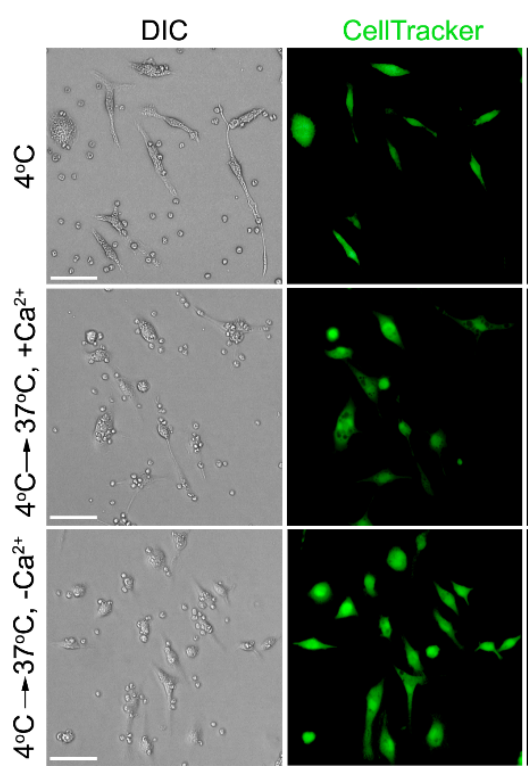

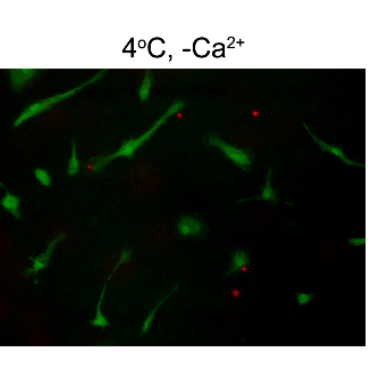
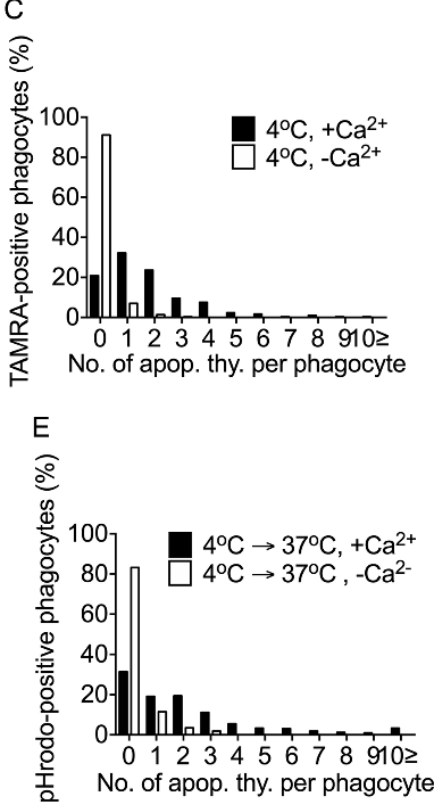

Figure 1. Extracellular calcium is necessary for internalization of apoptotic cells. (A) BMDMs treated with EGTA (10 mM) or cultured in calcium-free DMEM were incubated with TAMRA-stained apoptotic thymocytes for 30 min and analyzed by flow cytometry. TAMRA-positive BMDMs were considered to be phagocytes engulfing apoptotic cells. Control BMDMs incubated with apoptotic cells in DMEM containing calcium. $\mathrm{n}=3$ experiments, mean \pm SEM (one-way ANOVA). (B,C) CellTracker-stained BMDMs were incubated with TAMRA-labeled apoptotic thymocytes at $4{ }^{\circ} \mathrm{C}$ for $1 \mathrm{~h}$ in the presence or absence of calcium and observed by microscopy (B). The number of apoptotic cells bound to phagocytes was quantified (C). Scale bar, $50 \mu \mathrm{m} . \mathrm{n}=292\left(+\mathrm{Ca}^{2+}\right), 283\left(-\mathrm{Ca}^{2+}\right)$ cells. (D,E) CellTracker-stained BMDMs were incubated with pHrodolabeled apoptotic thymocytes at $4{ }^{\circ} \mathrm{C}$ for $1 \mathrm{~h}$, washed with PBS to remove unbound apoptotic thymocytes, and further incubated at $37^{\circ} \mathrm{C}$ for $30 \mathrm{~min}$ in the presence or absence of calcium (D). The number of pHrodo-positive BMDMs was quantified (E). Scale bar, $100 \mu \mathrm{m} . \mathrm{n}=400$ cells.

\subsection{Elevation of the Calcium Level in Phagocytes Is Due to Extracellular Calcium Entry during Efferocytosis}

The calcium level in phagocytes increases during efferocytosis. This is consistent with our extended observations, using various types of phagocytes, including professional and non-professional phagocytes and using Fura-2, a ratiometric dye (Figure 2A and S1A-D). Based on the finding that extracellular calcium is necessary for later stages of efferocytosis following the binding of apoptotic cells, elevation of the intracellular calcium level during efferocytosis may be due to extracellular calcium entry. However, other mechanisms, such as calcium release from intracellular stores and/or decreased calcium uptake by mitochondria, may underlie elevation of the intracellular calcium level. We first investigated whether decreased mitochondrial calcium uptake underlies elevation of the intracellular calcium level during efferocytosis, using Mdivi-1, which blocks mitochondrial fission through Drp-1 and thus promotes mitochondrial calcium uptake through the mitochondrial calcium uniporter (MCU) [30]. Mdivi-1 did not significantly alter the 
calcium level in BMDMs incubated without or with apoptotic cells (Figure 2B), suggesting that mitochondrial calcium flux is not a major contributor to elevation of the intracellular calcium level during efferocytosis. We next tested whether calcium release from the ER underlies elevation of the intracellular calcium level during efferocytosis, using 2-APB. It blocks $\mathrm{IP}_{3} \mathrm{R}$-mediated calcium release from the ER with an additional inhibitory effect on SOCE [31,32]. 2-APB abolished the increase in the calcium level in BMDMs incubated with apoptotic cells (Figure 2C and S2A), implying that calcium release from the ER likely is involved in elevation of the intracellular calcium level during efferocytosis. However, there is a possibility that the effect of 2-APB on the intracellular calcium level may be still caused by inhibiting SOCE in this experiment. Inhibition of $\mathrm{IP}_{3} \mathrm{R}$ can also block calcium entry into cells because calcium release from the ER activates CRACs and thus induces calcium entry through these channels. In addition, calcium may enter phagocytes through other channels, such as voltage-gated calcium channels during efferocytosis. To investigate this, we incubated phagocytes with apoptotic cells in calcium-free medium and measured the intracellular calcium level. The calcium level in BMDM incubated with apoptotic cells in the presence of extracellular calcium continuously increased, whereas this phenomenon was not observed in the absence of extracellular calcium (Figure 2D), suggesting that elevation of the intracellular calcium level during efferocytosis is due to extracellular calcium entry. We next tested which type of calcium channels is involved in the elevation of the intracellular calcium level using calcium channel blockers. YM58483 and SKF-96365, SOCE and CRAC inhibitors, nullified the effect of apoptotic cells on the calcium level in phagocytes, but nifedipine, a voltage-dependent calcium channel inhibitor, did not (Figure 2E and S2B). Noticeably, the effects of the inhibitors on elevation of the intracellular calcium level during efferocytosis corresponded to their effects on efferocytosis. 2-APB, YM58483, and SKF-96365 impeded efferocytosis, but nifedipine did not (Figure 2F and S2C,D). Interestingly, YM58483 abrogated efferocytosis but did not affect the binding of apoptotic cells to phagocytes (Figure $2 \mathrm{G}, \mathrm{H}$ ), supporting the idea that extracellular calcium entry is necessary for later stages of efferocytosis following the binding of apoptotic cells (Figure 1D,E). In summary, these data suggest that calcium release from the ER and extracellular calcium entry caused by this contribute to elevation of the intracellular calcium level and are necessary for efferocytosis.

\subsection{Apoptotic Cell Stimulation Induces the Orai1-STIM1 Interaction in Phagocytes}

Depletion of calcium from the ER is sensed by STIM1, which associates with and thereby activates Orai1, resulting in extracellular calcium entry through Orai1, a process called SOCE [19]. In addition, Orai1 and STIM1 are crucial for efferocytosis [22-24]. Thus, the association of Orai1 and STIM1 is likely modulated during efferocytosis. To investigate this, LR73 cells overexpressing STIM1 and Orai1 were stimulated with apoptotic cells or live cells and the Orai1-STIM1 association was monitored by an immunoprecipitation assay. Apoptotic cells increased Orai1-STIM1 association but not live cells (Figure 3A). This phenomenon was also observed at physiological protein levels. Orai1 and STIM1 associated more in BMDMs stimulated with apoptotic cells than in control BMDMs (Figure 3B). This increased association may be due to contamination of apoptotic cells. To investigate this and to observe the Orai1-STIM1 association in real time, we performed fluorescence resonance energy transfer (FRET) analysis. Orai1 and STIM1 C-terminally tagged with CFP and YFP, respectively, were introduced into LR73 cells and energy transfer was measured. FRET was $\sim 35 \%$ higher in phagocytes stimulated with apoptotic cells than in control phagocytes (Figure 3C,D, and Video S1). Noticeably, FRET measured using a microplate reader was increased in phagocytes incubated with apoptotic cells, but not in those incubated with living cells (Figure 3E). These data suggest that apoptotic cells induce the Orai1-STIM1 association in phagocytes during efferocytosis. 

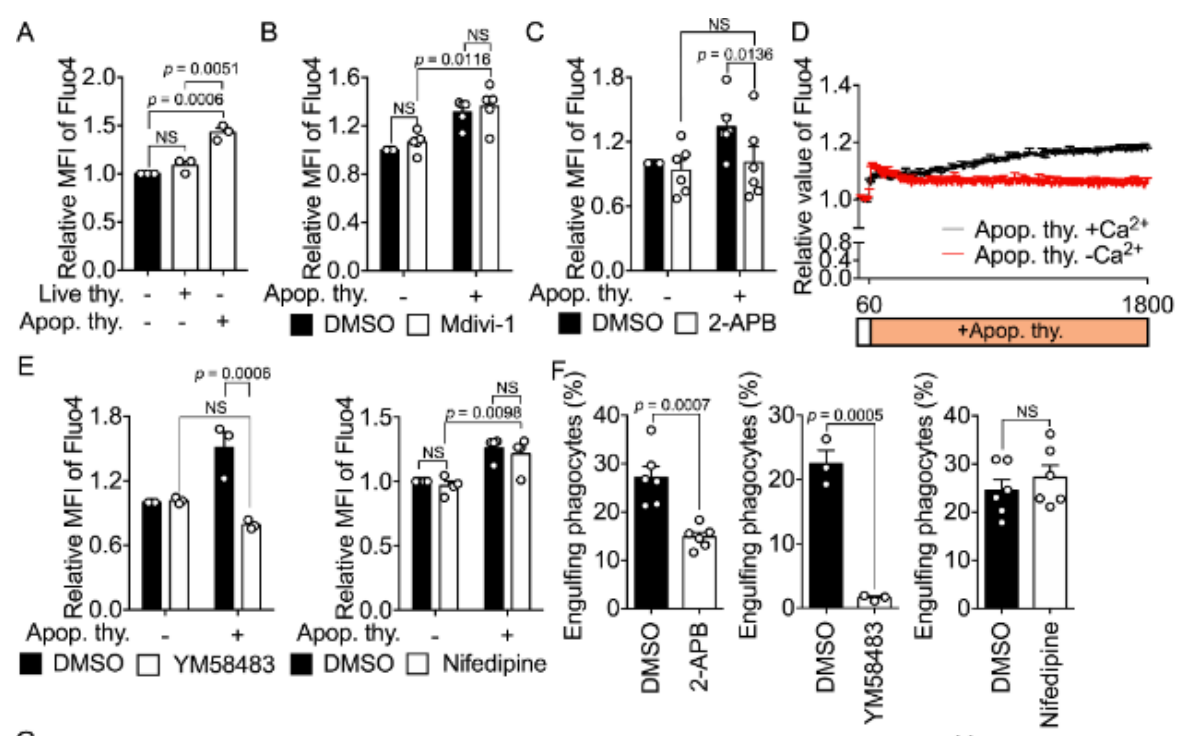

G

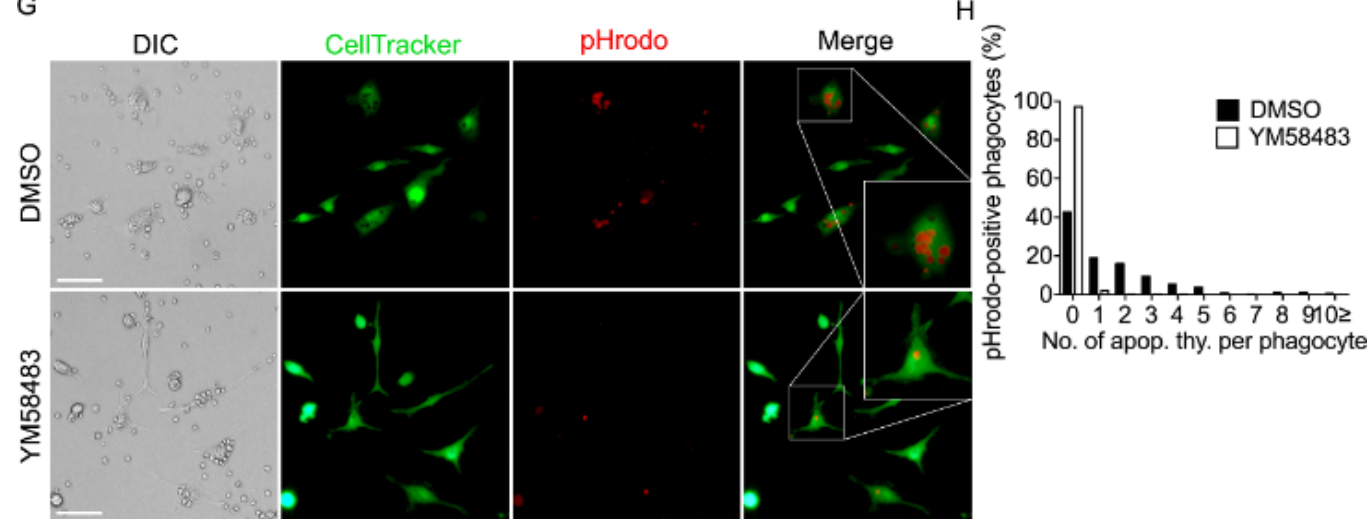

Figure 2. Elevation of the calcium level in phagocytes is due to extracellular calcium entry during efferocytosis (A) Fluo4-stained BMDMs were incubated with apoptotic thymocytes or live thymocytes and analyzed by flow cytometry. The MFIs of Fluo4 in BMDMs were compared. $\mathrm{n}=3$ experiments, mean \pm SEM (one-way ANOVA). (B,C) Fluo4-stained BMDMs were incubated with apoptotic cells in the presence or absence of Mdivi-1 $(10 \mu \mathrm{M})(\mathbf{B}, \mathrm{n}=5$ experiments $)$ or 2-APB $(200 \mu \mathrm{M})(\mathrm{C}, \mathrm{n}=6$ experiments). The MFIs of Fluo4 in BMDMs were measured by flow cytometry. Mean \pm SEM. NS, not significant (two-way ANOVA). (D) Fluo4-stained BMDMs were incubated with apoptotic cells in DMEM or calcium-free DMEM. The fluorescence intensity of Fluo4 in BMDMs was measured using FlexStation. $n=4$ experiments, mean \pm SEM. (E) Fluo-4-stained BMDMs were incubated with apoptotic cells in the presence or absence of YM58483 (100 $\mu \mathrm{M}, \mathrm{n}=3$ experiments) or Nifedipine ( $20 \mu \mathrm{M}, \mathrm{n}=4$ experiments) and analyzed by flow cytometry. Mean $\pm \mathrm{SEM}$ (two-way ANOVA). (F) BMDMs were incubated with TAMRA-stained apoptotic cells for $30 \mathrm{~min}$ in the presence or absence of 2-APB $(200 \mu \mathrm{M})$, YM58483 $(100 \mu \mathrm{M})$, or Nifedipine $(20 \mu \mathrm{M})$ and analyzed by flow cytometry. $\mathrm{n}=6$ (left), $\mathrm{n}=3$ (middle), and $\mathrm{n}=6$ (right) experiments, mean \pm SEM (two-tailed unpaired Student's $t$ test). (G,H) CellTracker-stained BMDMs were incubated with pHrodo-stained apoptotic thymocytes for $30 \mathrm{~min}$ in the presence or absence of YM58483 (100 $\mu \mathrm{M})$ and observed by microscopy $(\mathbf{G})$ and the number of pHrodo-positive apoptotic cells per phagocyte was quantified (H). Insets are en-largements of phagocytes engulfing apoptotic cells. Scale bar, $100 \mu \mathrm{M}$.

\subsection{Recognition of PS Is Necessary for Induction of the Orai1-STIM1 Association by Apoptotic Cells}

Next, we dissected the molecular mechanism by which apoptotic cells induce the Orai1-STIM1 association. First, we investigated whether apoptotic cells themselves or factors secreted by them induce the Orai1-STIM1 association. The Orai1-STIM1 association was not induced by apoptotic cell supernatants but was robustly induced by apoptotic cells (Figure 4A and S3A). This indicates that apoptotic cells themselves, but not molecules 
secreted by them, are responsible for induction of the Orai1-STIM1 association and that binding of apoptotic cells to phagocytes and/or subsequent stages of efferocytosis, such as internalization, and degradation of apoptotic cells are necessary for induction of this association. Next, to address which phase of efferocytosis is important for induction of the Orai1-STIM1 association, the internalization and degradation of apoptotic cells were blocked by cytochalasin $\mathrm{D}$, which inhibits actin polymerization, and bafilomycin $\mathrm{A}$, which impedes lysosomal acidification and thus degradation of cargos, respectively. Cytochalasin D and bafilomycin A appreciably impeded efferocytosis (Figure 4B,C). However, the Orai1-STIM1 association in phagocytes incubated with apoptotic cells in the presence of these inhibitors was comparable with that in control phagocytes (Figure 4D,E and S3B,C). This indicates that internalization and degradation of apoptotic cells are dispensable and binding of apoptotic cells to phagocytes is sufficient for induction of the Orai1-STIM1 association.

A
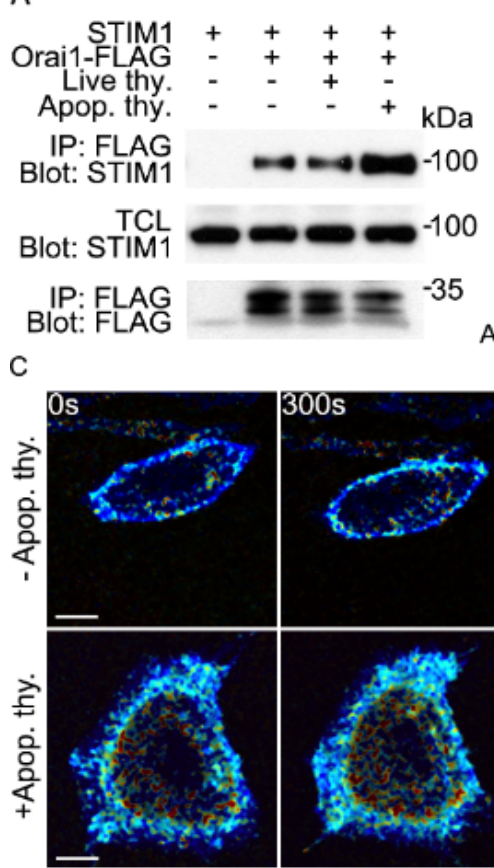

D
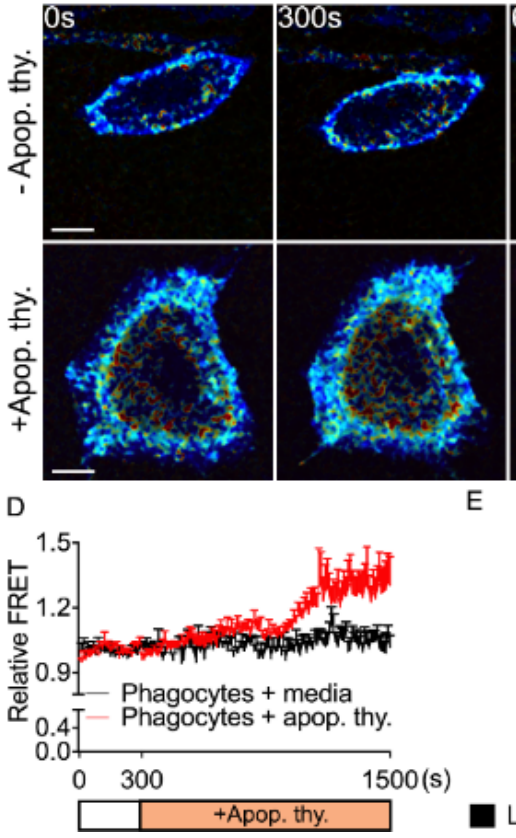

B

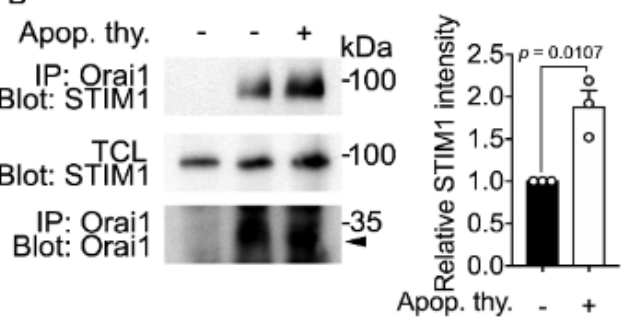

Live thy. - + Apop. thy. - - +
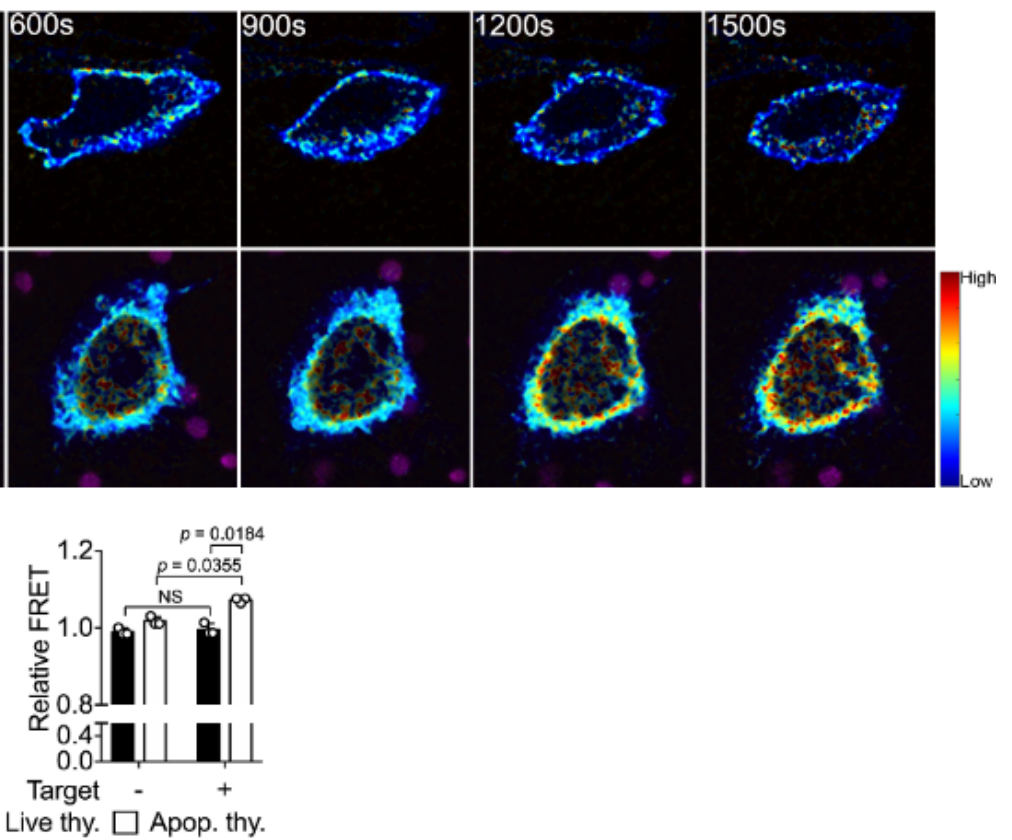

Figure 3. Apoptotic cell stimulation induces the Orai1-STIM1 interaction in phagocytes (A) LR73 cells were transfected with the indicated plasmids. After 1 day, cells were incubated with apoptotic thymocytes or live thymocytes for $10 \mathrm{~min}$ and lysed. FLAG-tagged Orai1 was immunoprecipitated with anti-FLAG antibody-conjugated agarose beads. Bound proteins were detected with the indicated antibodies (left) and co-immunoprecipitated STIM1 with Orai1 was quantified (right). The images are representative of three independent experiments. Mean \pm SEM (two-tailed unpaired Student's $t$ test). (B) Lysates of BMDMs stimulated with apoptotic cells for $10 \mathrm{~min}$ were incubated with an anti-Orai1 antibody and protein A/G agarose beads. Bound proteins were detected by immunoblotting (left) and co-immunoprecipitated STIM1 with Orai1 was quantified (right). The arrow head indicates Orai1. The images are representative of three independent experiments. Mean \pm SEM (two-tailed unpaired Student's $t$ test). (C,D) LR73 cells transfected with Orai1-CFP and STIM1-YFP were incubated with TAMRA-stained apoptotic cells for the indicated duration. FRET signals were observed by time-lapse confocal microscopy (C) and quantified (D). Images were obtained every $10 \mathrm{sec}$ for $30 \mathrm{~min}$. Scale bar, $10 \mu \mathrm{m} . \mathrm{n}=12$ (-Apop. thy) and $\mathrm{n}=13$ (+Apop. thy.) cells, mean \pm SEM. (E) LR73 cells transfected with Orai1-CFP and STIM1-YFP were stimulated with apoptotic or living thymocytes. FRET signals were detected using a microplate reader. $\mathrm{n}=3$ experiments, mean \pm SEM (two-way ANOVA). 
A

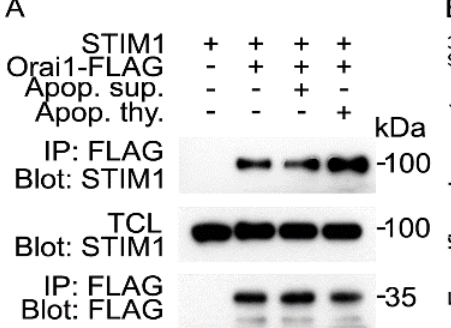

E

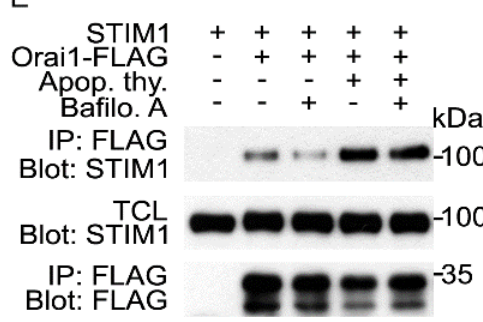

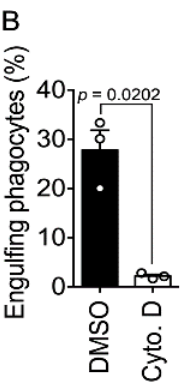

$\mathrm{F}$

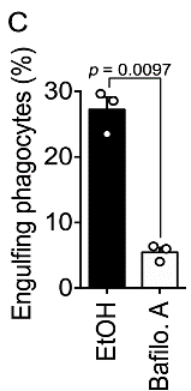

D

$\begin{array}{rllll}\text { STIM1 } & + & + & + & + \\ \text { Orai1-FLAG } & - & + & + & +\end{array}$

Apop. thy. - - + +

IP: FLAG Blot: STIM1

Blot: STIM1

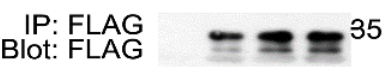

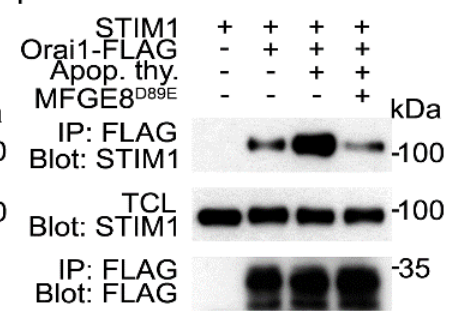

G

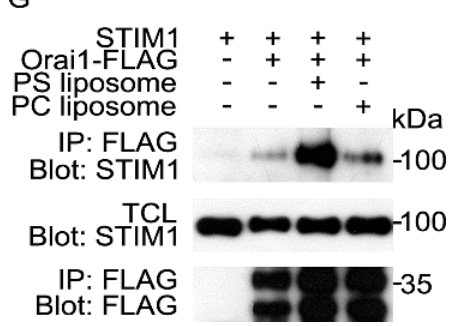

Figure 4. Apoptotic cell stimulation induces the Orai1-STIM1 association in a PS-dependent manner (A) LR73 cells transfected with the indicated plasmids were incubated with apoptotic cells or apoptotic cell supernatants for 10 min. Orai1-FLAG in cell lysates was precipitated with anti-FLAG antibody-conjugated agarose beads. Bound proteins were detected by immunoblotting. (B,C) LR73 cells were incubated with TAMRA-stained apoptotic cells for $2 \mathrm{~h}$ in the presence of the indicated concentration of cytochalasin $\mathrm{D}(1 \mu \mathrm{M})(\mathbf{B})$ or bafilomycin $\mathrm{A}(1 \mu \mathrm{M})(\mathbf{C})$, and engulfing phagocytes were analyzed by flow cytometry. $\mathrm{n}=3$ experiments, mean \pm SEM (two-tailed unpaired Student's $t$ test). (D-F) LR73 cells transfected with the indicated plasmids were stimulated with apoptotic cells for $10 \mathrm{~min}$ in the presence or absence of cytochalasin D $(1 \mu \mathrm{M})(\mathbf{D})$, bafilomycin $\mathrm{A}(1 \mu \mathrm{M})(\mathbf{E})$, or Mfge $8^{\mathrm{D} 89 \mathrm{E}}(\mathbf{F})$. The Orai1-STIM1 association was detected as in $(\mathbf{A})$. (G) LR73 cells transfected with Orai1 and STIM1 were stimulated with PC or PS liposomes for 10 min. Cell lysates were incubated with anti-FLAG antibody-conjugated agarose beads. Bound proteins were detected with the indicated antibodies. The images are representative of at least three independent experiments (A,D-G).

PS exposed on apoptotic cells is the best-known ligand to be directly or indirectly recognized by engulfment receptors on phagocytes. We thus tested whether PS is crucial for induction of the Orai1-STIM1 association during efferocytosis. To this end, PS on apoptotic cells was masked, using a Mfge8 mutant called Mfge ${ }^{\mathrm{D} 89 \mathrm{E}}$, which binds to PS on apoptotic cells but not to integrins on phagocytes [33], and the Orai1-STIM1 association was measured upon addition of PS-masked apoptotic cells. Apoptotic cells pretreated with purified Mfge ${ }^{\mathrm{D} 89 \mathrm{E}}$ failed to increase the Orai1-STIM1 association in phagocytes (Figure 4F and S3D). This finding was replicated when PS on apoptotic cells was masked by Anxa5, a PS-binding protein (Figure S4), suggesting that PS on apoptotic cells is necessary for induction of the Orai1-STIM1 association during efferocytosis. To further investigate whether PS is sufficient to induce the Orai1-STIM1 association, phagocytes were incubated with PS liposomes, a simplified mimic of apoptotic cells. The Orai1-STIM1 association was augmented in phagocytes incubated with PS liposomes but was unaltered in phagocytes incubated with phosphatidylcholine (PC) liposomes (Figure 4G and S3E). These data indicate that PS exposed on apoptotic cells is necessary and sufficient for induction of the Orai1-STIM1 association during efferocytosis. They further suggest that engulfment receptors that directly or indirectly recognize PS are upstream of signaling that induces the Orai1-STIM1 association.

\subsection{Mertk Is an Upstream Receptor of the PLC $\mathrm{C} 1-\mathrm{IP}{ }_{3} R$ Axis Activated by Apoptotic Cells}

A key signaling pathway for activation of Orai1 and induction of the Orai1-STIM1 association resulting in SOCE involves activation of PLC to cleave $\mathrm{PIP}_{2}$ into $\mathrm{IP}_{3}$ via G proteins or RTK cascades. $\mathrm{IP}_{3}$ then induces $\mathrm{IP}_{3} \mathrm{R}$-mediated calcium release from the ER, which triggers the Orai1-STIM1 association and calcium entry through Orai1 [34]. Thus, we tested whether the PLC $\gamma-\mathrm{IP}_{3} \mathrm{R}$ axis is activated during efferocytosis by measuring the 
phosphorylation levels of PLC $\gamma 1$ and IP ${ }_{3} R$. The levels of phosphorylated PLC $\gamma 1$ and IP ${ }_{3} R$ were higher in BMDMs incubated with apoptotic cells than in BMDMs incubated with live cells (Figure $5 \mathrm{~A}, \mathrm{~B}$ ), suggesting that the PLC $\gamma 1-\mathrm{IP}_{3} \mathrm{R}$ axis is activated during efferocytosis and that an engulfment receptor is upstream of this axis.
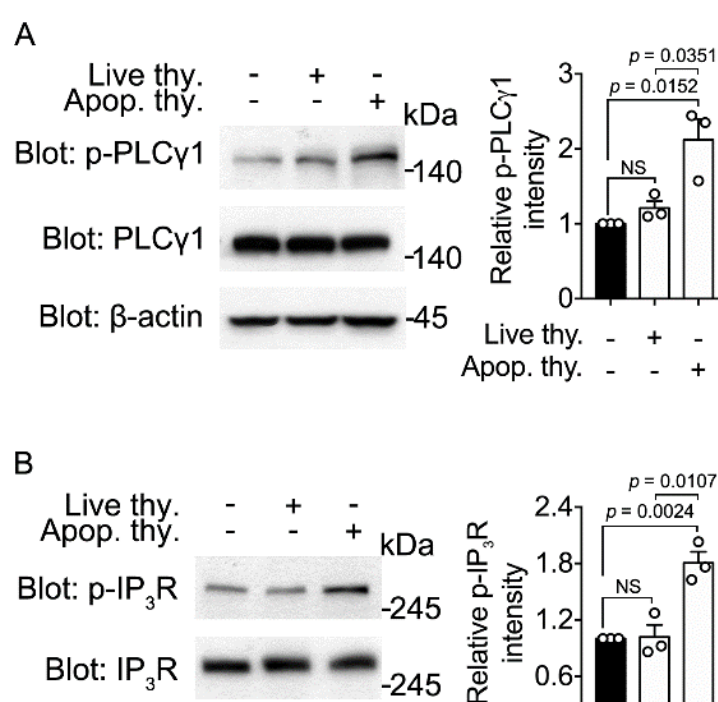

Blot: $\beta$-actin

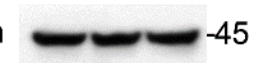

C
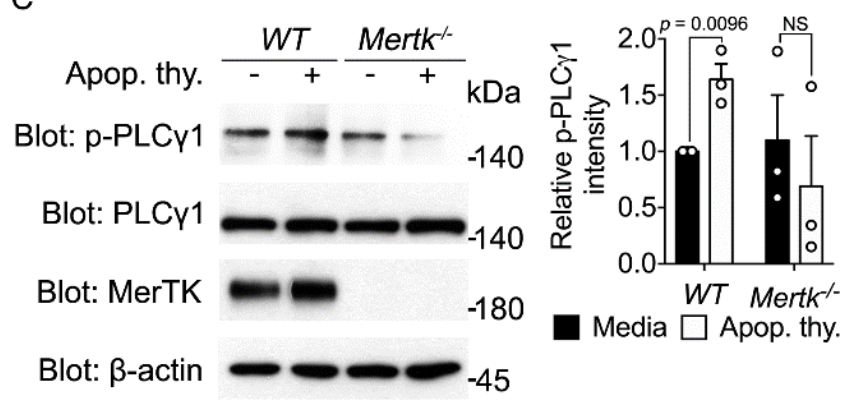

D
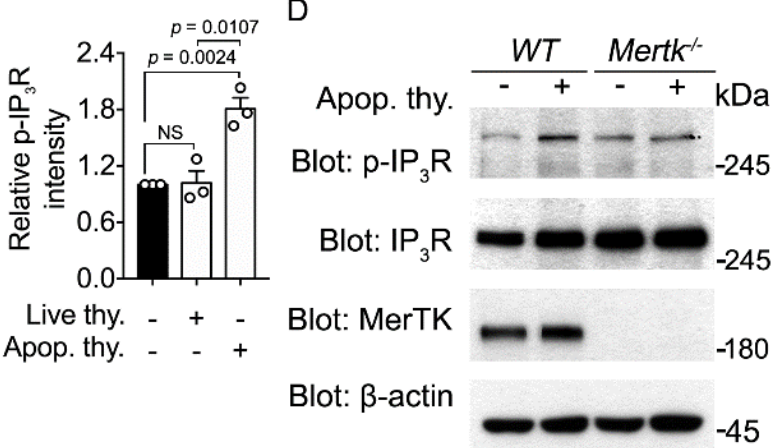

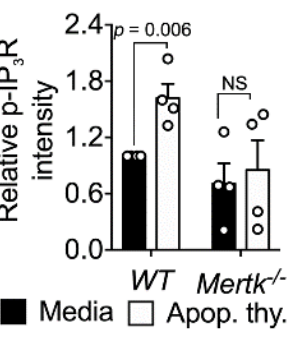

$-45$

Figure 5. Apoptotic cell stimulation activates the $\mathrm{PLC} \gamma 1-\mathrm{IP}_{3} \mathrm{R}$ axis $(\mathbf{A}, \mathbf{B}) \mathrm{BMDMs}$ were incubated with apoptotic thymocytes or live thymocytes for $10 \mathrm{~min}$ and lysed. Phosphor-PLC $\gamma 1$ (A) and phosphor-IP ${ }_{3} \mathrm{R}(\mathbf{B})$ in the lysates were detected by immunoblotting and quantified. $\beta$-actin was used as a loading control. The images are representative of three independent experiments. Mean \pm SEM (two-tailed unpaired Student's $t$ test). (C,D) BMDMs derived from Mertk ${ }^{-1-}$ and $W T$ mice were incubated with apoptotic cells for $10 \mathrm{~min}$ and lysed. Phospho-PLC $\gamma 1$ (C) and phosphor-IP ${ }_{3} R(D)$ in the lysates were detected by immunoblotting and quantified. The images are representative of three (C) or four (D) independent experiments. Mean \pm SEM (two-tailed unpaired Student's $t$ test).

Mertk is a member of the TAM receptor kinase family and also functions as an engulfment receptor that indirectly senses PS exposed on apoptotic cells through Gas6 and Pros [35]. In addition, PLC $\gamma 2$ is recruited to Mertk upon apoptotic cell stimulation [16]. Thus, Mertk may be an engulfment receptor upstream of the PLC $\gamma-\mathrm{IP}_{3} \mathrm{R}$ axis that induces the Orai1-STIM1 association during efferocytosis. To investigate this, the phosphorylation levels of PLC $\gamma 1$ and $\mathrm{IP}_{3} \mathrm{R}$ in BMDMs derived from Mertk ${ }^{-1-}$ and wild-type (WT) mice were compared upon apoptotic cell stimulation. In the basal state, the total and phosphorylation levels of PLC $\gamma 1$ and $\mathrm{IP}_{3} \mathrm{R}$ were comparable in Mertk ${ }^{-/}$and WT BMDMs. However, the phosphorylation levels of PLC $\gamma 1$ and $\mathrm{IP}_{3}$ R were substantially lower in Mertk $^{-/-}$BMDMs than in WT BMDMs upon incubation with apoptotic cells (Figure 5C,D), suggesting that Mertk is an upstream receptor of the $\mathrm{PLC} \gamma 1-\mathrm{IP}_{3} \mathrm{R}$ axis that induces the Orai1-STIM1 association.

\subsection{Mertk Depletion Attenuates the Orai1-STIM1 Association and Calcium Entry during Efferocytosis}

Next, to test whether Mertk functions as an upstream engulfment receptor that mediates the Orai1-STIM1 association during efferocytosis, this association was compared between Mertk ${ }^{-/}$and WT BMDMs upon apoptotic cell stimulation. Orai1 and STIM1 associated substantially less in Mertk ${ }^{-/-}$BMDMs than in WT BMDMs following apoptotic cell stimulation (Figure 6A). Next, we tested whether attenuation of the Orai1-STIM1 association in Mertk ${ }^{-/}$BMDMs upon apoptotic cell stimulation was coupled with the intracellular calcium level. The basal calcium level was comparable in $\mathrm{Mertk}^{-/-}$and 
WT BMDMs. However, apoptotic cell stimulation failed to increase the calcium level in Mertk $^{-/}$BMDMs (Figure 6B), suggesting that Mertk is an upstream receptor that elevates the intracellular calcium level during efferocytosis. We then tested whether the inability of apoptotic cell stimulation to increase the calcium level in Mertk $^{-/-}$BMDMs is due to alteration of SOCE. To this end, calcium in the ER was depleted by thapsigargin and calcium entry was monitored upon adding apoptotic cells. Intrinsic SOCE was indistinguishable between Mertk ${ }^{-/-}$and WT BMDMs. However, Mertk ${ }^{-/-}$BMDMs were unable to further increase SOCE upon apoptotic cell stimulation but WT BMDMs did (Figure 6C). SOCE, represented by the peak of Fluo4 fluorescence, was increased by 19\%, and the rate of calcium influx, as indicated by the slope (360-414 s), was also significantly increased in WT BMDMs. However, these phenomena were not observed in Mertk ${ }^{-/}$BMDMs upon apoptotic cell stimulation (Figure 6D,E), suggesting that Mertk is necessary for calcium entry during efferocytosis. Taken together, these results show that the Orai1-STIM1 association is induced through the PLC $\gamma 1-\mathrm{IP}_{3} \mathrm{R}$ axis downstream of Mertk, resulting in calcium entry and eventually elevation of the calcium level in phagocytes during efferocytosis.

A
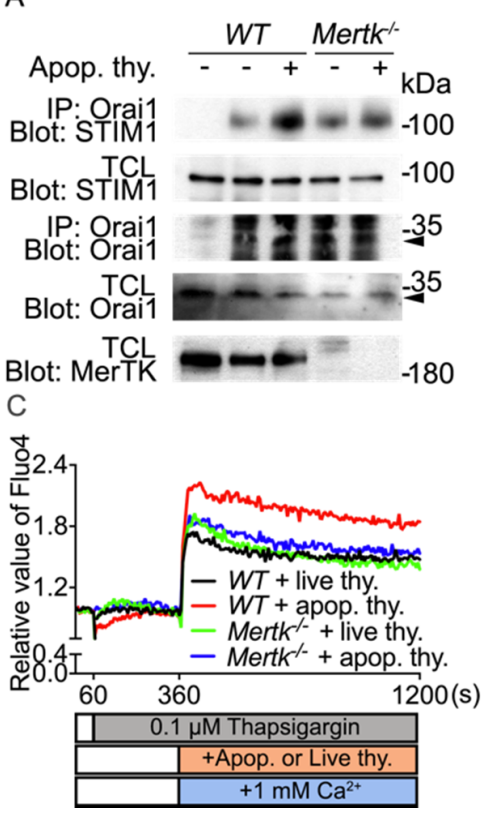

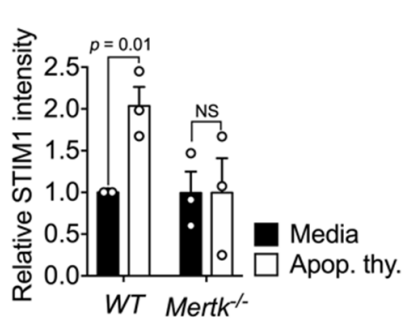

D

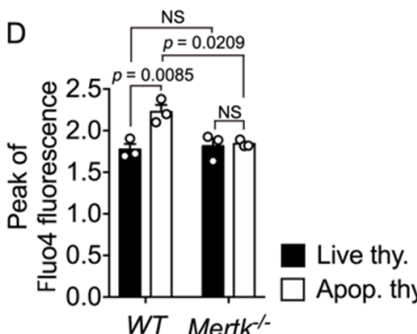

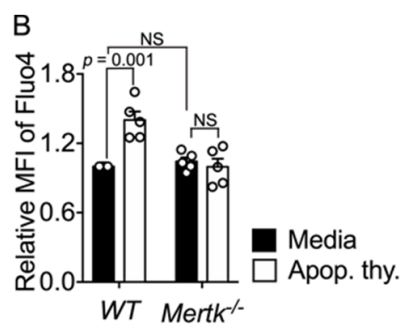

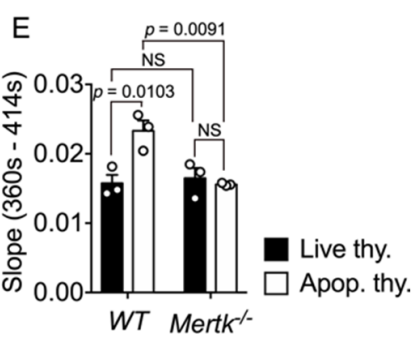

Figure 6. Mertk depletion attenuates the Orai1-STIM1 association and calcium entry (A) BMDMs derived from Mertk $^{-1-}$ and WT mice were incubated with apoptotic cells for $10 \mathrm{~min}$. Cell lysates were incubated with an anti-Orai1 antibody and protein A/G-conjugated agarose beads. Bound proteins were detected with the indicated antibodies (left) and co-immunoprecipitated STIM1 with Orai1 was quantified (right). The arrow heads indicate Orai1. The images are representative of three independent experiments. Mean \pm SEM (two-tailed unpaired Student's $t$ test). (B) BMDMs derived from Mertk ${ }^{-/-}$and WT mice were stained with Fluo4 and incubated with apoptotic cells. The MFIs of Fluo4 in the cells were analyzed by flow cytometry. $\mathrm{n}=5$ experiments, mean $\pm \mathrm{SEM}$ (two-way ANOVA). (C-E) BMDMs from the indicated mice were stained with Fluo4 and then treated with $0.1 \mu \mathrm{M}$ thapsigargin for the indicated duration. Thereafter, apoptotic or live thymocytes in medium containing $1.0 \mathrm{mM}$ calcium were added to the cells at the indicated time. Fluorescence of the cells was measured with a microplate reader. Data are representative of 4 independent experiments $(\mathbf{C})$, and the peak and slope of SOCE were calculated (D,E). $\mathrm{n}=3$ experiments, mean $\pm \mathrm{SEM}$ (two-way ANOVA).

\section{Discussion}

Efferocytosis is a sophisticatedly regulated process that specifically removes dying cells. Both intra- and extracellular calcium are indispensable for this essential process. In this study, we found that extracellular calcium enters phagocytes to increase the intracellular calcium level during efferocytosis, and this is mediated by the Orai1-STIM1 association 
induced by the Mertk-PLC $\gamma 1-\mathrm{IP}_{3} \mathrm{R}$ axis. A variety of engulfment receptors, including PS receptors, require calcium for binding to PS on apoptotic cells, and this may be one reason why extracellular calcium is necessary for efferocytosis. Our observations suggest that extracellular calcium also supplements intracellular calcium during efferocytosis. However, the roles of calcium in efferocytosis after it enters phagocytes remain unclear. Indeed, few studies have shown how calcium functions in efferocytosis. Recently, our group reported that Crbn, which is a component of the CRL4 ${ }^{\text {CRBN }}$ E3 ligase and regulates the level of Orai1, alters the amount of time required to internalize apoptotic cells [22]. Thus, one plausible role of calcium during efferocytosis is appropriate closure of the phagocytic cup to internalize apoptotic cells. We are currently studying the phases of efferocytosis in which calcium is involved.

The ER and mitochondria are intracellular organelles that store calcium [36]. A previous study reported that the upregulation of Drp-1 during efferocytosis increases mitochondrial fission, which blocks MCU-mediated mitochondrial calcium uptake and thus augments the intracellular calcium level [30]. Initially, we thought that this mechanism also augments the intracellular calcium level during efferocytosis. However, Mdivi-1 did not inhibit elevation of the intracellular calcium level, although it somewhat impeded efferocytosis. This discrepancy might be due to differences in the experimental conditions, such as the cell type used and the methods employed to measure the intracellular calcium level. Indeed, GCaMP6f, which was used as a calcium indicator in the previous study, may detect calcium more sensitively than Fluo4, which was used in this study. Thus, an inhibitory effect of Mdivi-1 on elevation of the intracellular calcium level might not have been detected. Nevertheless, we clearly showed that elevation of the intracellular calcium level upon apoptotic cell stimulation was abrogated in the presence of an CRAC inhibitor and in a calcium-free medium. This suggests that elevation of the intracellular calcium level during efferocytosis is mainly due to extracellular calcium entry and that diminished mitochondrial calcium uptake seems to only contribute marginally, if at all.

Apoptotic cell stimulation induces the Orai1-STIM1 association in a PS-dependent manner. The roles of Tim-4, a PS receptor, in efferocytosis are well-characterized [7,37]. Tim-4 cooperates with Mertk and integrins to phagocytose apoptotic cells [38-40]. Therefore, we analyzed the effect of Tim- 4 depletion on elevation of the intracellular calcium level upon apoptotic cell stimulation. In contrast with $\mathrm{Mertk}^{-/}$phagocytes, the intracellular calcium level was comparable in Tim- $4^{-/}$and WT peritoneal macrophages upon apoptotic cell stimulation (data not shown). This may be because Tim-4 does not mediate direct signaling [41,42] and the experiments were performed in the presence of serum, which enabled Mertk to sufficiently recognize apoptotic cells itself. In addition, we showed that intrinsic SOCE in Mertk ${ }^{-/}$and WT BMDMs is comparable. However, when SOCE was induced by $1 \mu \mathrm{M}$ thapsigargin and $2 \mathrm{mM}$ calcium, a typical condition to measure intrinsic SOCE, SOCE in Mertk ${ }^{-/-}$BMDMs was lower than in WT BMDMs. Apoptotic cell stimulation failed to increase SOCE in both WT and Mertk ${ }^{-/-}$BMDMs at this condition (data not shown). It may be because the thapsigargin concentration produces a stimulus inducing maximal SOCE. Nevertheless, the reason why intrinsic SOCE is lower in Mertk ${ }^{-/-}$ phagocytes than in WT phagocytes remains unclear at this condition and it would be interesting to investigate this in the future.

Collectively, the data presented in this study suggest that induction of the Orai1STIM1 association during efferocytosis increases the calcium level in phagocytes through SOCE and that Mertk is upstream of the PLC $\gamma 1-\mathrm{IP}_{3} \mathrm{R}$ axis responsible for induction of this association. Thus, our findings may help to comprehensively understand calcium flux in efferocytosis and to develop therapeutics for diseases associated with efferocytosis.

Supplementary Materials: The following are available online at https: / www.mdpi.com/article/ 10.3390/cells10102702/s1, Figure S1: Elevation of the calcium level in phagocytes during efferocytosis, Figure S2: The effects of 2-APB and SKF-96365 on efferocytosis and the calcium elevation in phagocytes, Figure S3: Quantification of immunoblots in Figure 4, Figure S4: Blocking PS on 
apoptotic cells attenuates Orai1-STIM1 association, Video S1: apoptotic cell stimulation induces Orai1-STIM1 interaction.

Author Contributions: Conceptualization, D.K. and D.P.; formal analysis, D.K., H.M., H.C., C.M., B.M., S.Y., J.L., S.-A.L. and H.P.; funding acquisition, D.P.; methodology, D.K., H.C., B.M. and D.P.; supervision, D.P.; validation, D.K., D.-H.L., D.J., G.L. and D.P.; writing-original draft, D.K. and D.P.; writing - review and editing, D.P. All authors have read and agreed to the published version of the manuscript.

Funding: This research was funded by the National Research Foundation of Korea funded by the Korea government (MSIP) (2019R1A2C1006480 and 2019R1A4A1028802) and by GIST Research Institute (GRI) ARI grant in 2021.

Institutional Review Board Statement: Not applicable.

Informed Consent Statement: Not applicable.

Data Availability Statement: Data supporting the findings of the study are available within the article and Supplementary Materials or from the corresponding author upon reasonable request.

Acknowledgments: We thank members of the Park laboratory at GIST for helpful discussions and critical reading of the manuscript.

Conflicts of Interest: The authors declare no conflict of interest. The funders had no role in the design of the study; in the collection, analyses, or interpretation of data; in the writing of the manuscript, or in the decision to publish the results.

\section{References}

1. Boada-Romero, E.; Martinez, J.; Heckmann, B.L.; Green, D.R. The clearance of dead cells by efferocytosis. Nat. Rev. Mol. Cell Biol. 2020, 21, 398-414. [CrossRef] [PubMed]

2. Lauber, K.; Blumenthal, S.G.; Waibel, M.; Wesselborg, S. Clearance of apoptotic cells: Getting rid of the corpses. Mol. Cell 2004, 14, 277-287. [CrossRef]

3. Fadok, V.A.; Voelker, D.R.; Campbell, P.A.; Cohen, J.J.; Bratton, D.L.; Henson, P.M. Exposure of phosphatidylserine on the surface of apoptotic lymphocytes triggers specific recognition and removal by macrophages. J. Immunol. 1992, 148, 2207-2216. [PubMed]

4. Fadok, V.A.; Bratton, D.L.; Frasch, S.C.; Warner, M.L.; Henson, P.M. The role of phosphatidylserine in recognition of apoptotic cells by phagocytes. Cell Death Differ. 1998, 5, 551-562. [CrossRef] [PubMed]

5. Penberthy, K.K.; Ravichandran, K.S. Apoptotic cell recognition receptors and scavenger receptors. Immunol. Rev. 2016, 269, 44-59. [CrossRef] [PubMed]

6. Park, D.; Tosello-Trampont, A.C.; Elliott, M.R.; Lu, M.; Haney, L.B.; Ma, Z.; Klibanov, A.L.; Mandell, J.W.; Ravichandran, K.S. BAI1 is an engulfment receptor for apoptotic cells upstream of the ELMO/Dock180/Rac module. Nature 2007, 450, 430-434. [CrossRef] [PubMed]

7. Miyanishi, M.; Tada, K.; Koike, M.; Uchiyama, Y.; Kitamura, T.; Nagata, S. Identification of Tim4 as a phosphatidylserine receptor. Nature 2007, 450, 435-439. [CrossRef]

8. Park, S.Y.; Jung, M.Y.; Kim, H.J.; Lee, S.J.; Kim, S.Y.; Lee, B.H.; Kwon, T.H.; Park, R.W.; Kim, I.S. Rapid cell corpse clearance by stabilin-2, a membrane phosphatidylserine receptor. Cell Death Differ. 2008, 15, 192-201. [CrossRef]

9. Doran, A.C.; Yurdagul, A., Jr.; Tabas, I. Efferocytosis in health and disease. Nat. Rev. Immunol. 2020, 20, 254-267. [CrossRef]

10. Lemke, G. Biology of the TAM receptors. Cold Spring Harb. Perspect. Biol. 2013, 5, a009076. [CrossRef] [PubMed]

11. Nakanishi, Y.; Shiratsuchi, A. Phagocytic removal of apoptotic spermatogenic cells by Sertoli cells: Mechanisms and consequences. Biol. Pharm. Bull. 2004, 27, 13-16. [CrossRef] [PubMed]

12. Feng, W.; Yasumura, D.; Matthes, M.T.; LaVail, M.M.; Vollrath, D. Mertk triggers uptake of photoreceptor outer segments during phagocytosis by cultured retinal pigment epithelial cells. J. Biol. Chem. 2002, 277, 17016-17022. [CrossRef]

13. Young, R.W.; Bok, D. Participation of the retinal pigment epithelium in the rod outer segment renewal process. J. Cell Biol. 1969, 42, 392-403. [CrossRef] [PubMed]

14. Lemke, G.; Burstyn-Cohen, T. TAM receptors and the clearance of apoptotic cells. Ann. N. Y. Acad. Sci. 2010, 1209, 23-29. [CrossRef] [PubMed]

15. Scott, R.S.; McMahon, E.J.; Pop, S.M.; Reap, E.A.; Caricchio, R.; Cohen, P.L.; Earp, H.S.; Matsushima, G.K. Phagocytosis and clearance of apoptotic cells is mediated by MER. Nature 2001, 411, 207-211. [CrossRef] [PubMed]

16. Todt, J.C.; Hu, B.; Curtis, J.L. The receptor tyrosine kinase MerTK activates phospholipase C gamma2 during recognition of apoptotic thymocytes by murine macrophages. J. Leukoc. Biol. 2004, 75, 705-713. [CrossRef] [PubMed]

17. Bagur, R.; Hajnoczky, G. Intracellular Ca ${ }^{2+}$ Sensing: Its Role in Calcium Homeostasis and Signaling. Mol. Cell 2017, 66, 780-788. [CrossRef] [PubMed] 
18. Felsenfeld, A.; Rodriguez, M.; Levine, B. New insights in regulation of calcium homeostasis. Curr. Opin. Nephrol. Hypertens. 2013, 22, 371-376. [CrossRef]

19. Prakriya, M.; Lewis, R.S. Store-Operated Calcium Channels. Physiol. Rev. 2015, 95, 1383-1436. [CrossRef]

20. Putney, J.W. Forms and functions of store-operated calcium entry mediators, STIM and Orai. Adv. Biol. Regul. 2018, 68, 88-96. [CrossRef]

21. Yeung, P.S.; Yamashita, M.; Prakriya, M. Molecular basis of allosteric Orai1 channel activation by STIM1. J. Physiol. 2020, 598, 1707-1723. [CrossRef]

22. Moon, H.; Min, C.; Kim, G.; Kim, D.; Kim, K.; Lee, S.A.; Moon, B.; Yang, S.; Lee, J.; Yang, S.J.; et al. Crbn modulates calcium influx by regulating Orai1 during efferocytosis. Nat. Commun. 2020, 11, 5489. [CrossRef]

23. Gronski, M.A.; Kinchen, J.M.; Juncadella, I.J.; Franc, N.C.; Ravichandran, K.S. An essential role for calcium flux in phagocytes for apoptotic cell engulfment and the anti-inflammatory response. Cell Death Differ. 2009, 16, 1323-1331. [CrossRef]

24. Cuttell, L.; Vaughan, A.; Silva, E.; Escaron, C.J.; Lavine, M.; van Goethem, E.; Eid, J.P.; Quirin, M.; Franc, N.C. Undertaker, a Drosophila Junctophilin, links Draper-mediated phagocytosis and calcium homeostasis. Cell 2008, 135, 524-534. [CrossRef]

25. Itoh, R.E.; Kurokawa, K.; Ohba, Y.; Yoshizaki, H.; Mochizuki, N.; Matsuda, M. Activation of rac and cdc42 video imaged by fluorescent resonance energy transfer-based single-molecule probes in the membrane of living cells. Mol. Cell. Biol. 2002, 22, 6582-6591. [CrossRef] [PubMed]

26. Min, C.; Park, J.; Kim, G.; Moon, H.; Lee, S.A.; Kim, D.; Moon, B.; Yang, S.; Lee, J.; Kim, K.; et al. Tim-4 functions as a scavenger receptor for phagocytosis of exogenous particles. Cell Death Dis. 2020, 11, 561. [CrossRef] [PubMed]

27. Stace, C.L.; Ktistakis, N.T. Phosphatidic acid- and phosphatidylserine-binding proteins. Biochim. Biophys. Acta 2006, 1761, 913-926. [CrossRef] [PubMed]

28. Santiago, C.; Ballesteros, A.; Martinez-Munoz, L.; Mellado, M.; Kaplan, G.G.; Freeman, G.J.; Casasnovas, J.M. Structures of T cell immunoglobulin mucin protein 4 show a metal-Ion-dependent ligand binding site where phosphatidylserine binds. Immunity 2007, 27, 941-951. [CrossRef] [PubMed]

29. Nakano, T.; Ishimoto, Y.; Kishino, J.; Umeda, M.; Inoue, K.; Nagata, K.; Ohashi, K.; Mizuno, K.; Arita, H. Cell adhesion to phosphatidylserine mediated by a product of growth arrest-specific gene 6. J. Biol. Chem. 1997, 272, 29411-29414. [CrossRef]

30. Wang, Y.; Subramanian, M.; Yurdagul, A., Jr.; Barbosa-Lorenzi, V.C.; Cai, B.; de Juan-Sanz, J.; Ryan, T.A.; Nomura, M.; Maxfield, F.R.; Tabas, I. Mitochondrial Fission Promotes the Continued Clearance of Apoptotic Cells by Macrophages. Cell 2017, 171, 331-345.e22. [CrossRef]

31. Saleem, H.; Tovey, S.C.; Molinski, T.F.; Taylor, C.W. Interactions of antagonists with subtypes of inositol 1,4,5-trisphosphate (IP3) receptor. Br. J. Pharmacol. 2014, 171, 3298-3312. [CrossRef] [PubMed]

32. Schild, A.; Bhardwaj, R.; Wenger, N.; Tscherrig, D.; Kandasamy, P.; Dernic, J.; Baur, R.; Peinelt, C.; Hediger, M.A.; Lochner, M. Synthesis and Pharmacological Characterization of 2-Aminoethyl Diphenylborinate (2-APB) Derivatives for Inhibition of Store-Operated Calcium Entry (SOCE) in MDA-MB-231 Breast Cancer Cells. Int. J. Mol. Sci. 2020, 21, 5604. [CrossRef] [PubMed]

33. Hanayama, R.; Tanaka, M.; Miwa, K.; Shinohara, A.; Iwamatsu, A.; Nagata, S. Identification of a factor that links apoptotic cells to phagocytes. Nature 2002, 417, 182-187. [CrossRef] [PubMed]

34. Bhardwaj, R.; Hediger, M.A.; Demaurex, N. Redox modulation of STIM-ORAI signaling. Cell Calcium 2016, 60, 142-152. [CrossRef]

35. Van der Meer, J.H.; van der Poll, T.; van't Veer, C. TAM receptors, Gas6, and protein S: Roles in inflammation and hemostasis. Blood 2014, 123, 2460-2469. [CrossRef]

36. Pozzan, T.; Rizzuto, R.; Volpe, P.; Meldolesi, J. Molecular and cellular physiology of intracellular calcium stores. Physiol. Rev. 1994, 74, 595-636. [CrossRef]

37. De Maeyer, R.P.H.; van de Merwe, R.C.; Louie, R.; Bracken, O.V.; Devine, O.P.; Goldstein, D.R.; Uddin, M.; Akbar, A.N.; Gilroy, D.W. Blocking elevated p38 MAPK restores efferocytosis and inflammatory resolution in the elderly. Nat. Immunol. 2020, 21, 615-625. [CrossRef]

38. Moon, B.; Lee, J.; Lee, S.A.; Min, C.; Moon, H.; Kim, D.; Yang, S.; Moon, H.; Jeon, J.; Joo, Y.E.; et al. Mertk Interacts with Tim-4 to Enhance Tim-4-Mediated Efferocytosis. Cells 2020, 9, 1625. [CrossRef]

39. Nishi, C.; Toda, S.; Segawa, K.; Nagata, S. Tim4- and MerTK-mediated engulfment of apoptotic cells by mouse resident peritoneal macrophages. Mol. Cell. Biol. 2014, 34, 1512-1520. [CrossRef]

40. Toda, S.; Hanayama, R.; Nagata, S. Two-step engulfment of apoptotic cells. Mol. Cell. Biol. 2012, 32, 118-125. [CrossRef]

41. Lee, J.; Park, B.; Moon, B.; Park, J.; Moon, H.; Kim, K.; Lee, S.A.; Kim, D.; Min, C.; Lee, D.H.; et al. A scaffold for signaling of Tim-4-mediated efferocytosis is formed by fibronectin. Cell Death Differ. 2019, 26, 1646-1655. [CrossRef] [PubMed]

42. Park, D.; Hochreiter-Hufford, A.; Ravichandran, K.S. The phosphatidylserine receptor TIM-4 does not mediate direct signaling. Curr. Biol. 2009, 19, 346-351. [CrossRef] 